as rodenticides and organochlorine, organophosphate and carbamate insecticides that targeted specific pests with broad-spectrum, nonspecific approaches. In addition to being toxic, many of these biocides had other biological effects such as mimicking hormones (see sidebar at right), suppressing immune systems or being concentrated in bodies of animals or humans.

Toxic pollutants from other sources (industrial and urban wastes and "leakage," public health, pest control, combustion byproducts, etc.) are also heavily responsible for losses of California's biodiversity in the past 4 to 5 decades. Toxic contaminants from these sources (such as PCBs, dioxins, heavy metals, sewage products, biocides used for public health and pest control, and many other materials) continue to "leak" into the environment and to exert negative effects on California's biota.

\section{Population declines, recoveries}

Another lesson that history taught us is that negative effects of biocides on biodiversity components are often not discovered until decades after a chemical's introduction, often after populations of organisms have been severely reduced or eliminated. While this used to be the rule rather than the exception, ecotoxicologists recognize that in the future, we will need to define or predict such problems before they occur rather than after (see box, p. 66). For example, literally before anyone knew what had happened, Peregrine falcons were extirpated in the entire eastern United States and were severely reduced in the rest of the country due to now-outmoded organochlorine insecticides. In almost all instances, the effects were directly attributable to the DDT metabolite DDE, which caused eggshell thinning, poor reproduction and subsequent population decline. Also involved were some "hard" (very toxic and persistent) organochlorine pesticides that caused direct mortality of adults. This is why many populations "crashed" - burning the candle at both ends, so to speak (both decreased fecundity and increased mortality occurred). It all

\title{
Unexpected side effects of chemicals acting as hormone mimics
}

\author{
D. Michael Fry
}

In addition to the toxic effects of pesticides and other environmental contaminants, some pollutants act like hormone mimics, causing disruption of the endocrine systems of fish and wildlife, and potentially, humans who might be exposed. These endocrine effects occur because the "foreign" chemicals bind to receptor molecules in hormone sensitive "target" cells (cells which respond to hormones, and produce effects related to the hormone action), and result in an abnormal response. Hormone disrupters are most injurious to developing embryos and to animals exposed during sensitive stages of the life cycle, such as during the breeding season.

Several examples of endocrine disruption in wildlife have been documented, including abnormal thyroid function in fish and birds exposed to PCBs and dioxins in the Great Lakes, testicular feminization of gulls exposed to DDT in their eggs, feminization of male fishes living downstream from municipal waste water discharge facilities, and male alligators with abnormally small penises living in a Florida lake contaminated with a variety of agricultural chemicals. A recent review by Colborn, et al. (1993) summarizes the information well.

Several pesticides, some industrial chemicals, and some natural plant compounds have estrogenic properties, that is they mimic the action of female steroid sex hormones. One chemical form of DDT, the "orthopara" isomer (designated o, $\mathrm{p}^{\prime}$-DDT) acts like an estrogen when it bioaccumulates in bird eggs, and causes male embryos to develop testes with partly ovarian characters, and to retain oviducts as in female birds. In southern California, the gull colonies exposed to DDT suffered population losses and an excess of breeding females, because feminized males did not reproduce, and did not even return to the colony.

Fish in some British rivers have been exposed to chemical effluents from municipal waste water treatment plants. Estrogenic alkyl-phenols (a group of industrial surfactants common in many household products) in the treated water are absorbed by the male fish, and the contaminants stimulate the male's livers to synthesize yolk proteins as if the male fish were going to lay eggs. This is a very recently discovered effect (White et al. 1994), and it is not yet known how common these pollutants may be. The effects, however, have prompted regulatory agencies to quickly begin to develop methods to screen for hormone disruptors, and to begin reassessing the regulations for testing chemicals and regulating waste discharge.

D. M. Fry is avian wildlife toxicologist, Department of Avian Sciences, U C Davis.

\section{For further reading}

Colborn, Theo, F.S. vom Saal, and A.M. Soto. 1993. Developmental Effects of EndocrineDisrupting Chemicals in Wildlife and Humans. Environmental Health Perspectives. 101: 37884.

White, R., S. Jobling, S.A. Hoare, J.P. Sumpter, and M.G. Parker. 1994. Environmentally persistent alkylphenolic compounds are estrogenic. Endocrinology. 135: 175-182 